\title{
SHAPING AND MANAGING SELF-IMAGE AND MODULAR IDENTITY 2.0 - THE CASE STUDY OF POLISH LANGUAGE WIKIPEDIA USERSPACE
}

\author{
Sebastian SKOLIK; Anna KARCZEWSKA; Katarzyna KUKOWSKA \\ Częstochowa University of Technology
}

\begin{abstract}
The user pages of Wikipedia enable self-presentation and are used to build a personal brand. Managing self-image is possible, among others, by small banners called userboxes. Initially, they were supposed to be objective information about someone, not an element of self-presentation of one's personality. However, different types of userboxes were introduced.

Purpose: The aim of the research is to analyze the use of the userboxes in the context of managing one's self-image and discussing the idea of modular identity 2.0.

Methodology: In the presented research, an attempt was made to characterize the course of the variability of userboxes as cultural elements that allow to shape and manage the image and determine the identity of the individuals posting them. For this purpose, qualitative and quantitative analysis of the content of the userboxes placed on the userpages was conducted, focusing primarily on the frequency of their occurrence and their modification over time.

Findings: The analysis of the content of over a thousand userboxes shows that after a short period of high variability at the beginning, the growth of new banners became smaller. As the results indicate, the most frequently replicated userboxes in Polish-language Wikipedia are those related to Wikipedia's digital environment, community identity and health. Moreover, the construction of self-image is becoming more and more reproductive, schematic and based on imitation.

Research limitations: Research has been conducted on one language version of Wikipedia. Some further comparisons with other language versions are recommended.
\end{abstract}

Key words: digital marketing, image management, personal brand, userboxes, Wikipedia Jel code: Z13

\section{Introduction}

In the digitalization era people more and more often build their self-image both in the real world and in the virtual space. They use various techniques connected with promotion which enable them to differentiate from other people. Personal branding is a new marketing concept related to the marketing strategies that people adopt in order to promote themselves in the market. People and their careers begin to be marked and perceived as brands. Personal branding is intended to create an asset as well as brand equity that refers to a particular person or individual. (Karaduman 2013).

Branding usually involves significant financial resources to create brand awareness and then subsequently brand loyalty. Social media enable people to promote themselves as brands in an efficient and usually cheap manner. The world

https://doi.org/10.11118/978-80-7509-820-7-0318

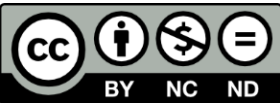


of business is beginning to acknowledge the importance of controlling personal brands and offer some advice on how to develop a personal brand identity through the use of different social media (Labrecque, Markos, Milne 2011).

In Wikipedia, the advertising ban is connected with one of its pillars, namely ,the neutral point of view". Apart from the encyclopedia's entry pages and special spaces constituting its technical backstage, userpages are an important part of the Wikipedia. To a limited extent, they are free from the prohibition of pushing through one's point of view, and thus also of presenting one's own system of values. This, in turn, is a gateway for promotional activities. One of ways of presenting self-image are banners called userboxes, which are the subject of this research.

The above remarks lead to considering the self-presentation of wikipedians and promoting their values in an environment which is critical to the promotional activity. In the text, the authors take up this thread in the context of the modularity of the content and the processes of imitation. The main aim of the research is to analyze the use of the userboxes in the context of managing one's own image and discussing the idea of modular identity 2.0, as one of the most important and interesting new trends in personal branding online.

\section{Literature review}

The userpages of Wikipedia enable self-presentation and are used to build a personal brand. D.L. Lair, K. Sullivan and G. Cheney (2005) describe personal branding as involving „concepts of product development and promotion (...) used to market persons for entry into or transition within the labor market". I. Shepherd (2005) in turn defined personal branding as a varied activities undertaken by individuals to make themselves known in the marketplace.

The first phase of personal branding is to establish own brand identity. People have to differentiate from the competition while fitting expectations of a specific target group. The second phase is to develop the brand's positioning by developing communication of one's brand identity through managing behavior, communication and use of symbols. The last phase is to evaluate a brand's image in order to fulfill personal and professional objectives (Khedher 2014, Khedher 2019).

Digital media facilitate innovation in self-presentation, but also generation of false identities. Creating fake accounts in social media is often used (sometimes permanently) in marketing activities. Creating own image on internet websites is therefore often treated suspiciously by other users in terms of authenticity of a given identity. Most digital media, both in the dotcom era and in the web 2.0 era, are driven by advertising revenues and in this respect are no different from the traditional media. Users are attacked by marketing messages, but they are also involved in promoting products and services (Shafieea et al. 2020).

Wikipedians often use their user pages to present themselves, their values, skills, and project activities. The design of the user's website and content, as long as they 
do not exceed the acceptable standards, can be shaped freely. A significant number of wikipedians' userpages are based on template patterns that have spontaneously developed in the project. Managing one's self- image is possible, among others, by the userboxes. They are small banners which the users put on their user pages (user profiles) (fig. 1). They are banners about hobbies, interests, place of residence, popular culture, sexual orientation and political affiliation. Creating a userpage does not require using them, but a significant number of people do it, expressing their own characteristics. Often for this purpose, the userboxes are „borrowed” from other users. In this way, the range of the most attractive ones is increased.

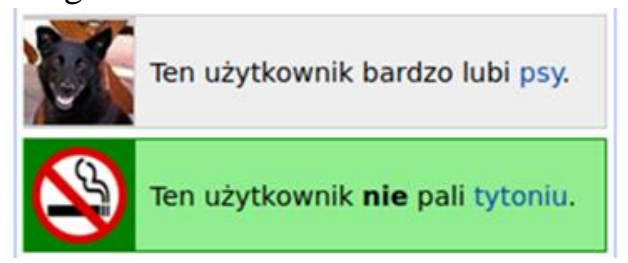

Figure 1. Examples of userboxes

Source: pl.wikipedia.org; the first picture by Pleple2000, CC-BY-SA-3.0

Modularity seen in webpage creation can be described in the context of psychoevolutionary and memetic theories. The diversity of the identities of the individuals results from the multiplicity of modules at the disposal of their minds (Buss 2003, pp. 70-78). J. Neckar points out, however, that the modular nature of mind is not necessarily what evolutionary psychologists portray. „Modularity in terms of cognitive, motivational and emotional functions, perhaps, should be interpreted more as sensitivity to a certain type of environmental impact and readiness for a certain direction of development, and not as a largely complete structure" (Neckar 2018, p. 185). In memetics, modularity refers to the cultural evolution. It occurs by replicating and mutating its tiniest elements, the memes, in peoples' minds. Contrary to evolutionary built-in modules, memes are acquired in the process of socialization, yet the effectiveness of their replication depends also on their adaptation to the mental environment of an individual (Łaszczyca 2017). The analyzes of the processes of co-creating cultural content in the cyberspace raise the issue of the importance of modularity in the context of possibility of changing (mixing) and copying (replicating) previously shared works. Wikipedia is cited there as a major project which value is increasing due to small improvements made by thousands of internet users.

The first userboxes were created to present one's knowledge of languages. Hence, it was supposed to be objective information about someone, not an element of selfpresentation of one's own personality. However, other types of userboxes were introduced very quickly. In the English version of Wikipedia, these were banners about hobbies, interests, place of residence, popular culture, sexual orientation and political affiliation (Westerman 2009). 
Promoting certain values, attitudes and world views through the wikipedian's userpages is usually not a strictly marketing activity, but it is building self-image and managing it by manipulating the components of the website. The higher the social status of a wikipedian (resulting from his activity and obtained rights), the greater the content he presents can be accepted by the community. At the same time, along with creating their own identity as a wikipedian, the users try to promote the values of the project to a greater extent (see: Neff et al. 2013). The presentation of nonneutral values (political, religious) is also tolerated to a greater extent, if they are common among the members of a given language version of Wikipedia.

The wikipedian's userpage, as not being part of the encyclopedia, allows for more free posting of content and also a more liberal approach to the issue of credibility. Hence, committed wikipedians often create controversial userboxes. In some language versions it has even become necessary to establish general rules for creating this type of banners (Westerman 2009). Credibility and neutrality are among Wikipedia's guiding principles for creating Wikipedia content.

Research on discussions on the deletion of individual articles in the English version of Wikipedia indicates that the course of these discussions is especially lively when they are joined by people who post a lot of information about themselves on their own userpages (Joyce and Pike 2017). Research on the political identity of wikipedians implies that the issue of identity change is connected with the transition from engaging in editing articles significant for the individual to acting for Wikipedia as a whole. And thereby, peripheral units move to the center and begin to engage in the community life (Neff et al. 2013).

\section{Methodology}

In the presented research, an attempt was made to characterize the course of the variability of userboxes as textual and graphic cultural elements allowing to determine the identity of the entities publishing them in the Polish-language Wikipedia. For this purpose, an analysis of the content of the pages of users posting userboxes was performed, focusing primarily on the frequency of their occurrence, their modification over time and their subject.

Since the userboxes with their own pages are most often categorized, the PetScan tool was used to generate a list of all the userboxes located in the category tree assigned to this type of pages. In this way, the number of 2223 userbox pages and their ID numbers were obtained, which allowed for easier determination of the increase in the diversity of userboxes over time. Each created page gets subsequent ID number. Out of this number, 1078 userboxes were selected for further analysis, which were considered susceptible to changes as they did not refer to formalized information on Wikipedia editing skills.

The further stages of the undertaken research were: determining which userboxes are the result of modification of other banners, examining the range of variability (the number of derived userboxes), the degree of dissemination on wikipedians' 
userpages and their survival rate. The last element of the study was limited to checking how the number of userboxes on the userpages of the most active wikipedians' changes over time. In this sense, the survival does not mean the very existence of userbox pages, but their potential „contagiousness”. The long-term participant observation conducted by one of the authors of this text allows us to state that very often copying of userboxes takes place through imitation.

\section{Results and discussion}

One of the most common ways to distribute userboxes is through the process of learning how users edit Wikipedia. If the user is familiar with the basic elements of the code but is technically unable to edit, he often watch the changes made by others. In the same way, wikipedians also „borrow” elements of userpages from themselves. The more often a given type of userbox is used, the greater the likelihood of multiple exposure of the mind to this visual stimulus. Usually, the wikipedians publish a whole set of userboxes in one edition on their userpage. However, authors of new variants of userboxes produce them over longer periods of time, even if it is a series of similar banners.

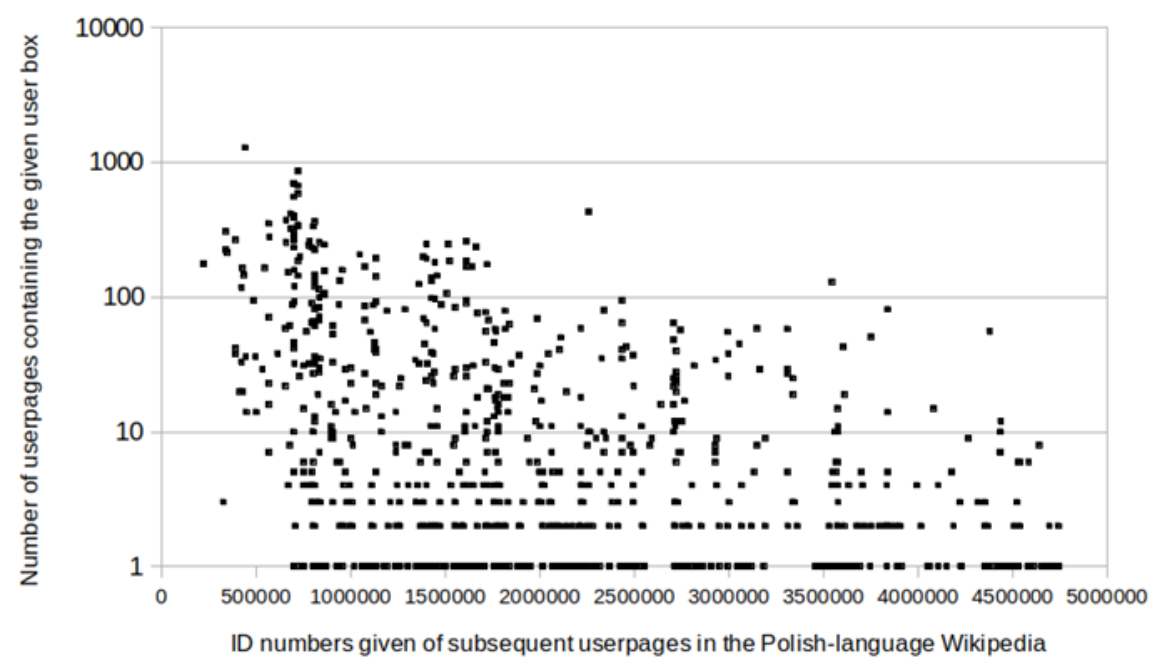

Figure 2. Distribution of the appearance of subsequent userboxes over time in the Polish-language Wikipedia. $N=913$

Source: Own research

The first userboxes in the Polish-language Wikipedia appeared in 2005, and their rapid growth took place in 2006-2009. Among the analyzed userboxes susceptible to changes, more than half were produced in this period. The time of the creation of a given userbox is also important in the context of the multiplication of its copies. In the case of banners created before 2010, as many as 76 were placed on 100 or more userpages. Of the boxes created later, only 2 were placed on over 100 userpages. 
Around 165 userboxes were never used on the wikipedians userpages, including 70 created by the end of 2009 (12.4\%) and 95 created later (18.6\%). There are 28.3 copies per userbox out of the 1078 pool. The distribution of the frequency of userboxes on wikipedians's userpages is presented in figure 2 .

It was also determined how the number of userboxes changed over time among the most active wikipedians. For this purpose, the lists of the 600 most active users published at the beginning of 2007, at the end of 2011 and at the beginning of 2020 were compared. The activity concerns the number of editions made by a given account from the beginning of the activity until the time of counting.

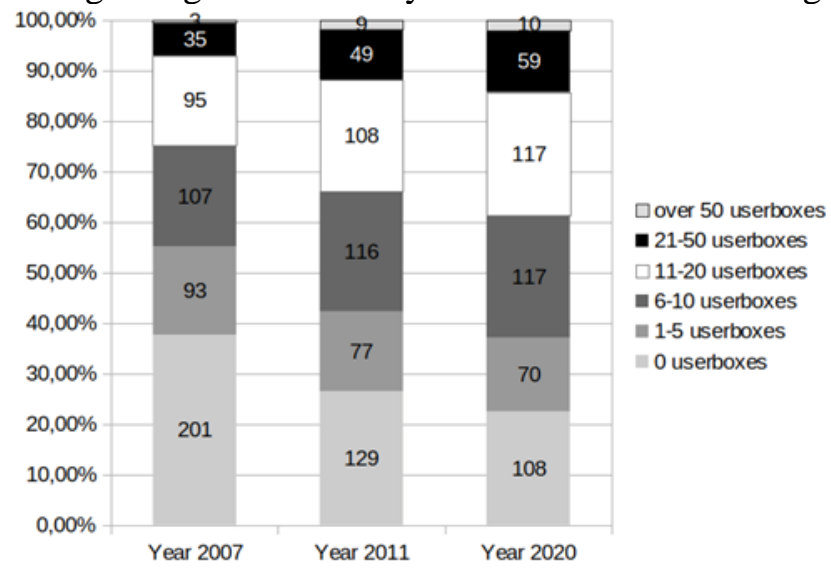

Figure 3. Changes in the number of userboxes on the userpages of the most active wikipedians. Generated on the basis of the lists on the Wikipedia website: Najaktywniejsi wikipedyści

Source: Own research

The increase in the number of wikipedians userpages containing userboxes and the increase in the number of userboxes in general was much greater between 2007 and 2011 than between 2011 and 2020 (fig. 3).

In order to be able to determine the degree of variability, the content was categorized. In terms of the text used, userboxes were divided into several categories. The first category concerns the activity of a wikipedian. It includes the userboxes defining action (123), experience (84), competences (94) and being a user of certain website, software, etc. (157). The next category included userboxes defining the wikipedian's relationship to the outside world: judgments about something (18), interest in something (17), emphasizing one's belonging to a place/community, etc. (125), pointing to being a supporter of something (282) or an opponent of something (31). The third category included 134 userboxes that explicitly defined the identity of an individual („I am X”), the fourth one included 5 userboxes that were abstract jokes. In addition, 8 userboxes were personalized pieces of the interface helpful in building the "tower". As the results indicate, the most frequently replicated userboxes in Polish-language Wikipedia are those related to Wikipedia's digital environment, community identity and health. 
19 userboxes were created by modifying one of the official banners, while 49 others were derived from them. 142 userboxes were classified as original (not a modification of previous userboxes), which gave rise to another 388, including 331 in the second generation, 43 in the third, and 10, 3 and 1 respectively. For 480 userboxes, no derivative banners were identified.

In the case of derived userboxes, it was most often a simple modification, consisting in changing one or two elements, especially when they were created by the same user. Serial creation of userboxes with minimal modifications is visible especially after 2008, which indicates a decline in the formation of new niches. A parallel process is progressive standardization which makes it easier to evaluate the contribution of Wikipedians and the quality of the produced encyclopedia content.

The analysis allowed to identify the userbox elements subject to modifications. These include: text modification by rewriting, changing the link and content, changing the meaning, shortening or extending the description; modification of the graphic content or the letter code of the userbox (changing the graphics to a different one, using modified graphics, changing the case and font size in the letter code); modification of the color of the text background, graphics background (letter code) or the frame of the userbox.

In the analyzes of contemporary media, it can be noticed that long narratives are replaced by increasingly shorter statements - tweets, news tickers in television programs or visual simplifications. N. Postman assumes that in this way word-based culture is replaced with iconographic culture (Postman 2002). This is favored by the remix culture developed thanks to free licenses (Lessig 2014). This culture allows for the collective creation of encyclopedia entries, while at the same time sharing the code and modifying other people's works. This characteristics are observed also while analyzing the self-image building by the use of userboxes.

The most frequently replicated userboxes in Polish-language Wikipedia were related to Wikipedia's digital environment, community identity and health, while W. Westerman (2009) indicated that userboxes concerned mainly hobbies, interests, place of residence, popular culture, sexual orientation and political affiliation. These subjects also appear in the userboxes used by Polish Wikiedists. Frequently duplicated is also the content that is humorous and refers to emotions.

In spite of the fact that there is a high level of imitation in the use of userboxes, for many users it is not a thoughtless action. This is evidenced by the self-ironic and critical nature of using the userboxes. For example, due to the frequent use of userboxes informing about the consumption of various types of beverages, a humorous banner stating that „this user is drinking denatured alcohol” appeared.

Using the userboxes on the userpages serves to make and promote own personal brand and at the same time help to stand out from, but also to identify with the Wikipedia community. The content of the userpages detail and support the significant element of the personal brand which is a username of the wikipedians (Karaduman 2013). However, using the name of the institution or enterprises in order to advertise oneself in the Wikipedia is prohibited in Polish-language Wikipedia. 


\section{Conclusion}

The users of the Polish-language Wikipedia, similarly to other language versions, use special banners - userboxes, to introduce themselves to other members of this project. Although recognition is achieved thanks to own username, the userboxes allow you to build your ,uniqueness". With time, however, an increasing number of wikipedians use userboxes created by others, often copying sets of userboxes onto their own userpages. This may be due to the smaller technical competences of newcomers in the subsequent ,generations" of wikipedians.

Research has shown a clear tendency to imitate in the use of userboxes. The most popular are those that were created in the first few years. Moreover, over time, another userboxes were created as modifications of the existing ones.

Unlike social media, Wikipedia's own pages are hardly used to build a personal brand. Even though wikipedians devote a certain amount of time to create their selfimage, the level of activity in the project is still more important to their prestige. It is recommended to verify the results of this study in other language-versions of Wikipedia to extrapolate conclusions to a wider community.

\section{References}

1. Buss D.M. (2003), Psychologia ewolucyjna, GWP, Gdańsk.

2. Joyce E., Pike J. (2017), Too Much Information: The Influence of User Self-Presentation on Success in Mass Collaboration, In: 23rd Americas Conference on Information Systems Boston.

3. Karaduman I. (2013), The effect of social media on personal branding efforts of top level executives, „Procedia - Social and Behavioral Sciences”, 99, pp. 465-473.

4. Khedher M. (2014), Personal Branding Phenomenon, „International Journal of Information, Business and Management”, 6, 2, pp. 29-40.

5. Khedher M. (2019), Conceptualizing and researching personal branding effects on the employability, „Journal of Brand Management”, 26, 2, pp. 99-09, DOI:10.1057/s41262-0180117-1

6. Labrecque L.I., Markos E., Milne G.R. (2011), Online Personal Branding: Processes, Challenges, and Implications, „Journal of Interactive Marketing”, 25, pp. 37-49, DOI:10.1016/j.intmar.2010.09.002

7. Lair D.L., Sullivan K., Cheney G. (2005), Marketization and the recasting of the professional self: The rhetoric and ethics of personal branding, „Management Communication Quarterly”, 18, 3, pp. 307-343, DOI:10.1177/0893318904270744

8. Lessig L. (2014), Remix: making art and commerce thrive in the hybrid economy, Penguin Books, New York.

9. Łaszczyca P. (2017), Memy w pamięci: jak wyśledzić memy z mózgu, „Teksty z Ulicy. Zeszyt Memetyczny", 18, pp. 11-42.

10. Neckar J. (2018), Ewolucyjna psychologia osobowości, Wydawnictwo Akademickie Sedno Warszawa.

11. Neff J.J., Laniado D., Kappler K.E., Volkovich Y., Aragón P., Kaltenbrunner A. (2013), Jointly They Edit: Examining the Impact of Community Identification on Political Interaction in Wikipedia, „PLoS ONE”, 8(4): e60584, DOI:10.1371/journal.pone.0060584

12. Postman N. (2002), Zabawić się na śmierć, Muza S.A., Warszawa. 
13. Shafiee M., Gheidi S., Sarikhani Khorrami M., Asadollah H. (2020), Proposing a new framework for personal brand positioning, ,European Research on Management and Business Economics", 26, 1, pp. 45-54, DOI:10.1016/j.iedeen.2019.12.002

14. Shepherd I. (2005), From Cattle and Coke to Charlie: Meeting the Challenge of Self Marketing and Personal Branding, „Journal of Marketing Management”, 21, 5/6, pp. 589606, DOI: 10.1362/0267257054307381

15. Westerman W. (2009), Epistemology, the Sociology of Knowledge, and the Wikipedia Userbox Controversy, In: Blank T.J. (ed.), Folklore and the Internet: Vernacular Expression in a Digital World, pp. 135-158, Utah State University Press, Logan. 